\title{
BMJ Open Meta-analysis of the effectiveness of psychological and medical treatments for binge-eating disorder (MetaBED): study protocol
}

\author{
Anja Hilbert, ${ }^{1}$ David Petroff, ${ }^{2}$ Stephan Herpertz, ${ }^{3}$ Anette Kersting, ${ }^{4}$ \\ Reinhard Pietrowsky, ${ }^{5}$ Brunna Tuschen-Caffier, ${ }^{6}$ Silja Vocks, ${ }^{7}$ Ricarda Schmidt ${ }^{1}$
}

To cite: Hilbert A, Petroff D, Herpertz S, et al. Metaanalysis of the effectiveness of psychological and medical treatments for binge-eating disorder (MetaBED): study protocol. BMJ Open 2017;7: e013655. doi:10.1136/ bmjopen-2016-013655

- Prepublication history and additional material is available. To view please visit the journal (http://dx.doi.org/ 10.1136/bmjopen-2016013655).

Received 28 July 2016 Revised 23 January 2017 Accepted 31 January 2017

CrossMark

For numbered affiliations see end of article.

\section{Correspondence to} Professor Anja Hilbert; anja.hilbert@medizin.unileipzig.de

\section{ABSTRACT}

Introduction: Binge-eating disorder (BED) was included as its own diagnostic entity in the Fifth Edition of the Diagnostic and Statistical Manual of Mental Disorders (DSM-5). An increasing number of treatment studies have been published, but an up-todate comprehensive meta-analysis on diverse treatment approaches for BED is lacking. In an updated and extension of a previous meta-analysis, the goals of this study are to assess the short-term and long-term effectiveness of psychological and medical treatments for BED.

Methods and analysis: We will search bibliographic databases and study registries, including manual searches for studies published before January 2016. The search strategy will include terms relating to binge eating and diverse forms of psychological and medical interventions. Language will be restricted to English. The studies included will be treatment studies, that is, randomised-controlled trials, and non-randomised and non-controlled studies, for individuals with BED (DSM-IV or DSM-5), and studies that provided a pre-treatment and at least one post-treatment or follow-up assessment of binge eating. The primary outcomes will be the number of binge-eating episodes, abstinence from binge eating and diagnosis of BED at post-treatment and/or follow-up(s), and changes from pre-treatment to post-treatment and/or follow-up(s). Likewise, as secondary outcomes, eating disorder and general psychopathology, quality of life, and body weight will be analysed and adverse events and treatment drop-out will be examined. Study search, selection and data extraction, including risk of bias assessment, will be independently performed by 2 reviewers and consensus will be sought. Moderator analyses will be conducted, and equity aspects will be considered. Sensitivity analyses will be conducted to determine the robustness of the results.

Ethics and dissemination: Ethical approval is not required for this meta-analysis. Published in a peerreviewed journal and disseminated electronically and in print, this meta-analysis will form the basis of the renewal of the German evidence-based S3 Guidelines of Diagnosis and Treatment of Eating Disorders, specifically BED.

Trial registration number: CRD42016043604.

\section{Strengths and limitations of this study}

- Strengths of this study are the provision of a comprehensive meta-analysis of the efficacy and effectiveness of psychological and medical treatments for binge-eating disorder, including identification of moderators of outcome.

- The PRISMA-P and MOOSE guidelines for meta-analysis protocols are followed.

- GRADE factors will be considered for evaluating the quality of evidence.

- A limitation is that economic aspects will not be considered.

\section{BACKGROUND}

Binge-eating disorder (BED), characterised by recurrent binge eating that occurs in the absence of regular inappropriate compensatory behaviours, was first included as its own diagnostic entity in the Fifth Edition of the Diagnostic and Statistical Manual of Mental Disorders (DSM-5). ${ }^{1}$ Compared with its provisional inclusion as an eating disorder diagnosis in need of further study in the preceding version of the DSM (DSM-IV), ${ }^{2}$ the DSM-5 requires binge-eating episodes to occur once per week over 3 months, lowering the diagnostic threshold of the DSM-IV, which required at least 2 days of binge eating per week over 6 months. For both diagnostic classifications, extant literature has indicated BED to be associated with severe health impairments, including increased eating disorder and general psychopathology, mental comorbidity, obesity and associated medical sequelae, and impaired quality of life. With a lifetime prevalence rate of $1.9 \%, \mathrm{BED}$ is the most common eating disorder, with peak onset in adolescence or early adulthood. ${ }^{3} 4$ An increasing number of clinical studies investigating the efficacy (explanatory trials to determine whether an intervention produces the expected effect under ideal, highly 
controlled circumstances) and effectiveness (pragmatic trials to measure the effect under 'real world', less controlled conditions) ${ }^{5}$ of diverse treatment approaches to BED has been published and compiled in meta-analyses $^{6-13}$ and systematic ${ }^{6} \quad{ }^{14-19}$ as well as narrative reviews. ${ }^{20-22}$

Among the meta-analyses, which in contrast to reviews allow a quantitative comparison of effects, two comprehensive reports addressed the efficacy and effectiveness of several broader treatment categories of BED, for example, psychotherapy, self-help treatment, weight loss treatment, pharmacotherapy and combined treatments. Vocks et $\mathrm{l}^{13}$ analysed 38 treatment studies for individuals with BED (randomised-controlled trials (RCTs), and non-randomised and non-controlled studies), until June 2006. In examining the RCTs, they found that psychotherapy and structured self-help, both mostly based on cognitive-behavioural therapy, significantly reduced binge eating (large effects) and eating disorder psychopathology (medium-to-large effects) at post-treatment when compared with untreated control groups. RCTs on pharmacotherapy, mainly using antidepressants, significantly reduced binge eating at post-treatment (medium effect), but not the associated eating disorder psychopathology (less than small effects), in comparison with untreated control groups. Both psychotherapy and pharmacotherapy significantly reduced post-treatment depressive symptoms (medium effects). Uncontrolled studies on conservative weight loss treatment demonstrated a significant post-treatment reduction of binge eating (medium effect), and a non-significant reduction of depressive symptoms. Combination treatments did not yield larger effects than monotherapeutic approaches. Except for weight loss treatment (medium effect), none of the interventions resulted in a significant weight reduction. In the RCTs, drop-out rates were nonsignificant in psychotherapy, self-help and pharmacotherapy when compared with untreated control groups. Important limitations of this early meta-analysis consist of a lack of examination of adverse effects and safety, quality of life, risk of bias and a lack of systematic documentation of the search process.

Recently, a comprehensive systematic review and meta-analysis of 34 psychological and pharmacological RCTs for BED searched up to November 2015 (for MEDLINE up to May 2016) provided several meta-analytic comparisons with regard to waiting list or placebo control conditions, showing that therapist-led cognitive-behavioural therapy led to a greater posttreatment abstinence from binge eating when compared with waiting list. Furthermore, second-generation antidepressants were superior to placebo at post-treatment in bringing about abstinence from binge eating and reducing binge-eating episodes, eating disorder psychopathology and depression, but not weight. ${ }^{6} 8$ The stimulant lisdexamfetamine showed significantly greater odds of achieving post-treatment abstinence from binge eating and a greater reduction of binge-eating episodes and eating disorder psychopathology when compared with placebo. Serious adverse events were documented for lisdexamfetamine. Further meta-analytic comparisons on other treatment approaches, for example, structured self-help or weight loss treatment, were not conducted. With a concentration on RCTs, the effectiveness of treatments under 'real-world' conditions was not addressed.

Other meta-analyses specifically focused on one category of treatment only, for example, pharmacotherapy, ${ }^{12}$ specifically antidepressants ${ }^{11}$ or lisdexamfetamine,${ }^{23} \mathrm{com}-$ bination treatments, ${ }^{24}$ or manualised self-help, ${ }^{7}$ while providing detailed results for BED alone. A further meta-analysis on RCTs in bulimia nervosa and recurrent binge eating, ${ }^{9}{ }^{10}$ however, did not specify the results for $\mathrm{BED}$ as its own diagnosis.

Since Vocks et $a l^{13}$ conducted their meta-analysis (search until June 2006), a number of large-scale clinical studies of BED and/or their long-term follow-ups have been published. ${ }^{25-27}$ Adding these studies would increase the precision of effect estimation; allow for the examination of longer term follow-up effects in order to determine the maintenance of therapeutic gains over time and facilitate moderator analyses with sufficient power. Further planned extensions of Vocks et $a l^{13}$ include an examination of adverse effects associated with treatment in order to evaluate safety; evaluation of quality of life; use of standard tools of risk of bias assessment and inclusion of other treatments with measured effect on BED symptomatology (eg, bariatric surgery). In the planned meta-analysis, a comprehensive search strategy based on the largest databases and clinical registries and a systematic documentation of the search in a PRISMA flow diagram will be performed. The PRISMA-P ${ }^{28}$ and MOOSE $^{29}$ guidelines for reporting of the meta-analysis will be followed.

\section{Objectives}

The objectives of this meta-analysis are: to assess the short-term and long-term effectiveness of psychological and medical treatments for patients with BED in randomised-controlled, non-randomised and noncontrolled treatment studies with inactive or active control groups, regarding binge eating, eating disorder and general psychopathology, quality of life, and body weight; to determine adverse events and treatment drop-out; and to examine equity aspects and risk of bias (PICO framework; Population, Intervention, Comparison, Outcome). Following this procedure, this report is intended to form the basis of the renewal of the German evidence-based S3 Guidelines of Diagnosis and Treatment of Eating Disorders ${ }^{30}$ specifically BED, aimed at guiding the translation of clinical research into practice.

\section{METHODS AND ANALYSIS}

\section{Study designs}

Randomised-controlled treatment studies will be included, complemented by non-randomised and non-controlled 
studies for increasing external validity. Case reports and studies with a sample size smaller than $n=10$ will be excluded.

\section{Participants}

Patients with a pre-treatment diagnosis of BED according to DSM-IV or DSM-5, including BED of low frequency and/or limited duration, will be included. Studies examining multiple patient groups will be included in case of separate data reports for patients with BED.

\section{Experimental interventions}

Psychological (eg, psychotherapy, self-help, conservative weight loss treatment) and medical treatments (eg, pharmacotherapy, bariatric surgery) applied to individuals with BED are included in this study. Treatments are defined to be attempts to relieve or cure BED or a highly related comorbidity (eg, bariatric surgery for treatment of obesity).

\section{Control conditions}

Inactive control groups (eg, no treatment, wait-list, placebo) and active control groups (eg, time/attention control, usual care, other treatment) will be considered as control conditions.

\section{Outcomes}

The core pathology of BED (binge-eating episodes or days, abstinence from binge eating, and/or diagnosis of BED) is to be assessed as an outcome measure. Sufficient data are required to allow the calculation of effect sizes (eg, M, SD and/or n, \% at pre-treatment and post-treatment or follow-up(s)). Thus, a pre-treatment and at least one post-treatment or follow-up assessment are necessary.

Primary outcomes: As primary outcomes, the number of binge-eating episodes, abstinence from binge eating and diagnosis of BED will be considered, forming the main outcome criteria in most treatment studies of BED. ${ }^{13}$ Binge-eating episodes commonly involve eating an amount of food that is definitely larger than what other people would eat under similar circumstances, associated with a sense of loss of control (LOC) over eating (objective binge-eating episodes). ${ }^{131}$ As bariatric surgery limits the possible amount of food intake, ${ }^{32}$ episodes of LOC eating which include both objective and subjective binge-eating episodes (eating an objectively or subjectively large amount of food, associated with a sense of LOC over eating), are considered where appropriate. Abstinence from binge eating will be defined as zero binge-eating episodes over a specified time frame, and presence or absence of a diagnosis of BED will be based on DSM-IV or DSM-5.

Secondary outcomes: As secondary outcomes, associated eating disorder psychopathology will be operationalised through attitudes regarding eating behaviour and body image, for example, as determined through the Eating Disorder Examination or the Eating Disorder Inventory.
Further, general, non-eating disorder psychopathology will be operationalised through measures of depression, for example, the Beck Depression Inventory or Hamilton Rating Scale for Depression. Quality of life will be operationalised through measures focusing on mental and/or physical well-being, for example, the Short Form Health Survey or the Impact of Weight on Quality of Life Scale. Body weight, body mass index (BMI, $\mathrm{kg} / \mathrm{m}^{2}$ ) or other measures of excess weight will be considered if derived from objective measurement of body weight and height. Potential adverse consequences of the treatment will be operationalised as total adverse events and adverse events related to the intervention. Drop-out from treatment will be determined as a proxy of compliance with treatment.

\section{Search methods}

The search strategy will include terms related to binge eating and diverse forms of psychological and medical interventions in title, abstract and keywords (or full texts): (binge eat*) AND (efficac* OR effect* OR outcome OR counsel* OR interven* OR pharmaco* OR drug OR psychoanaly* OR psychotherap* OR therap* OR treat* OR train* OR weight loss OR weight reduction OR self-help OR bariatric surg* OR weight loss surg* OR weight reduction surg* OR obesity surg*; for a full search example see online supplementary file).

Language restrictions will refer to English. Studies published or accepted for publication from inception to January 2016 will be sought. The searches will be rerun before the final analyses and further studies retrieved for inclusion prior to submission of the meta-analytical report. A new search for the total publication time period will be carried out because of greater availability of databases and registries when compared with Vocks et $a l^{13}$ and because of the necessity to systematically document the search in a PRISMA flow diagram.

The following data sources will be used:

1. Electronic bibliographic databases: MEDLINE, PubMED, PsycINFO, EMBASE, PUBPSYCH, LILACS, CINAHL, AMED, Web of Science, DARE, ANNUAL REVIEWS, NIHR Centre for Reviews and Dissemination, CDSR, Clinical Psychology Review.

2. Study registries: PROSPERO, CENTRAL, International Clinical Trials Registry Platform, ClincalTrials.gov, EU Clinical Trials Register, ISRCTN Trial Registry, Deutsches Register Klinischer Studien. Authors of ongoing studies will be contacted.

3. Manual searches: reference lists of included studies and review articles identified during the search. Publications in the International Journal of Eating Disorders from 1990 to January 2016.

\section{Study selection}

Two psychologists (MSc level) will independently review all abstracts and titles for eligibility. If deemed eligible or where eligibility is unclear, full-text papers will be obtained. Disagreement will be resolved through 
consensus. The two psychologists will independently assess all full-text papers for inclusion. Where unclear because of a lack of information, study authors will be contacted. Disagreement will be resolved through consensus and under supervision of AH. In case of additional publications referred to in the primary included paper (eg, study protocol, intervention description) these publications will be obtained and considered as part of the included study. Multiple reports within the framework of one study will be assembled in order to form one unit of analysis in the review. Double publications of the same trial will be excluded, based on automatic and manual screening.

\section{Data extraction}

The standardised coding scheme and handbook used by Vocks $e t a l^{13}$ with evidence of good inter-rater reliability will be extended and updated with regard to this meta-analysis' goals. The handbook provides definitions, coding instructions, examples and an overview of data management. Data extraction will be performed independently by two psychologists (MSc level). While one rating will include the entire study information, the duplicate rating will concentrate on outcome variables and further essential information to be used in meta-analytic comparisons as well as risk of bias assessment. ${ }^{33}$ Following training, a pilot procedure will be conducted using a representative sample of 10 studies to be reviewed independently. Disagreement between raters will be resolved through consensus and in consultation with AH. Data collection refers to: eligibility, study design, inclusion and exclusion criteria, participant characteristics (eg, sociodemographic, anthropometric), time points of assessment, sample size, intervention characteristics (eg, setting, duration, compliance, integrity), outcomes, drop-out, and adverse events, and risk of bias (eg, blinding, sequence generation, allocation sequence concealment). Data will be recorded for pretreatment, post-treatment and/or follow-up, where available. In order to facilitate comparisons with Vocks et $a l,{ }^{13}$ the previous ratings will be compared with the current ratings by determining inter-rater reliability for the main outcome variables.

\section{Risk of bias assessment}

The Cochrane Collaboration's Risk of Bias Tool ${ }^{33}$ and the Effective Practice and Organization of Care Risk of Bias Tool $^{34}$ will be used to assess the risk of bias in randomised-controlled studies as well as non-randomised studies, with additional items for assessing the risk of bias in uncontrolled studies, for example, considering the newly published Risk of Bias in Non-randomized Studies of Interventions (ROBINS-I). ${ }^{35}$ Summary assessments of low, medium or high risk of bias will be performed as recommended..$^{33}$ The risk of bias will be examined during data extraction, following the procedure of independent coding and disagreement resolution described above, and will be used to interpret outcome data.

\section{Equity}

Participant characteristics known to be important from an equity perspective will be recorded (PROGRESS framework; Place, Race, Occupation, Gender, Religion, Education, Socioeconomic status, Social status). ${ }^{36}$ In addition, information will be collected on whether or not interventions included particular strategies to address diverse or disadvantaged populations. If indicated, these characteristics will be used in moderator analyses (see Moderation analyses section).

\section{Statistical models and measures of treatment effect}

Two types of analyses will be performed. First, the treatment effect will be compared for treatment versus noninterventional and placebo controls. These analyses will be performed for each treatment category separately (see below), and only RCTs will be included. Second, the treatment effect will be estimated within each treatment category, where all types of studies will be included. Random-effects models (general and generalised linear mixed models) will be used and compared with fixed-effects models as a sensitivity analysis.

Primary outcomes: The number of binge-eating episodes (or days) over the past 28 days will be coded. If the time period differs from 28 days, the number of episodes will be scaled appropriately. The treatment effect will be measured as a standardised mean difference between the number of binge-eating episodes at post-treatment and/or follow-up(s) and the number at baseline. The CI associated with the treatment effect relies on the SD of this difference. For estimation of this SD, the following procedure will be used:

1. If available, $\mathrm{SD}$ will be taken directly from the article; otherwise,

2. If a test without covariates for the difference in question was performed, then SD will be determined from the test statistic or $p$ value; otherwise,

3. The SD for the difference in means will be estimated from the SD at baseline and the SD at post-treatment, assuming a correlation between the two based on available data.

If none of the above is available, the trial will not be included in the analysis of this primary outcome. If only median and IQR are provided, mean and SD will not be estimated, since high abstinence rates at post-treatment may result in a median of 0 , making such estimates unreliable. The above outcome will be complemented by the difference in means, since binge eating episodes are all measured on the same scale and the result can be easier to interpret.

Abstinence from binge eating and diagnosis of BED are binary outcomes and will be measured as rates at post-treatment (after attempting to account for different observational times as necessary). The Wilson CI will be used to quantify uncertainty on the estimate and rates will be analysed for each treatment category separately. A comparison of intervention to control groups for RCTs will use ORs. 
Secondary outcomes: Continuous and binary outcomes will be treated along similar lines as specified for the primary outcomes above. If different scales are used, standardised means will be computed. For count data, if counts are small enough that treating them as proportions (ie, a continuous variable) may be inappropriate, then tables containing the count (numerator) and the number of patients (denominator) will be provided.

\section{Unit of analysis}

Treatment groups within publications will be the units of analysis both for randomised and non-randomised trials.

\section{Dealing with missing data}

As a sensitivity analysis, the subset of trials will be analysed where there has been appropriate treatment of missing values (eg, multiple imputations). Such a sensitivity analysis may not be needed if the subset differs only from the entire set or is very small.

\section{Assessment of heterogeneity}

The standard statistic in meta-analyses, $\mathrm{Q}$, will be used to quantify and test how much individual trials deviate from the grand mean. Moreover, the variance $\tau^{2}$ from the random-effects model will be provided.

\section{Assessment of reporting biases}

Funnel plots with standardised mean differences on the horizontal and SE on the vertical axis will be inspected. As sensitivity analyses, trim and fill procedures and the fail-safe $\mathrm{N}$ will be used to quantify how unpublished negative outcome trials might affect results.

\section{Moderation analyses}

A power analysis is planned. Various parameters can be expected to affect outcomes and will be treated with meta-regression if the number of trials permits. Characteristics describing the trials will be presented in tables. The following variables will be extracted:

- Treatment characteristics: format (eg, individual, group), treatment category (eg, psychotherapy, selfhelp treatment, weight loss treatment, pharmacotherapy, bariatric surgery and combined treatments), single treatments (eg, cognitive-behavioural therapy, specific drugs), length of treatment.

- Participant characteristics: recruitment, age, sex, ethnicity, education, pre-treatment values (eg, baseline number of binge-eating episodes, BMI), duration of BED, diagnosis of BED.

- Study design: RCT, non-randomised trial with comparison group, non-randomised trial without comparison group, type of blinding (eg, patient, outcome assessors).

- Analysis method: for example, intention-to-treat analyses, completer analyses.

- Manualisation: for example, manual used, no manual use reported.
- Treatment integrity check: for example, integrity check by means of therapist supervision, no integrity check used.

- Therapist training: for example, general training of therapists, specific training for the study intervention, no training of therapists.

- Interview-based assessment of binge eating and/or of BED: for example, structured clinical interview (eg, Eating Disorder Examination), no structured clinical interview.

\section{Strength of evidence}

An evaluation of the strength of evidence will be provided with consideration of the GRADE recommendations.

\section{Study record and data management}

Records retrieved from electronic database and manual searches will be managed using EndNote X7 (Thomson Reuters, 2013). Data extraction of selected studies will be performed using a predefined Microsoft Excel (Microsoft Office Professional Plus 2010, V.14.0.7015.1000) coding form based on the coding scheme (see Data extraction section). All data will be analysed using R V.3.3.0 (R Core Team. R: A language and environment for statistical computing. Vienna, Austria: R Foundation for Statistical Computing 2016. http://www.R-project.org/).

\section{DISSEMINATION}

The study results will be disseminated through peerreviewed publication (electronic and in print) and conference presentations to the scientific community, and through further publications and presentations to the public and healthcare professionals. This meta-analysis is intended to form the basis of the renewal of the German evidence-based S3 Guidelines of Diagnosis and Treatment of Eating Disorders, ${ }^{30}$ specifically BED, guiding the dissemination of evidence-based treatments into clinical practice.

No restrictions on publication exist. Authorship will follow the Rules of Good Scientific Practice of the German Research Foundation, and no professional writers will be used.

\section{Author affiliations \\ ${ }^{1}$ Department of Medical Psychology and Medical Sociology, Department of Psychosomatic Medicine and Psychotherapy, University of Leipzig Medical Center, Leipzig, Germany \\ ${ }^{2}$ Clinical Trial Center Leipzig, University of Leipzig, Leipzig, Germany ${ }^{3}$ Department of Psychosomatic Medicine, LWL-University Clinic, Ruhr- University Bochum, Bochum, Germany \\ ${ }^{4}$ Department of Psychosomatic Medicine and Psychotherapy, University of Leipzig Medical Center, Leipzig, Germany \\ ${ }^{5}$ Department of Clinical Psychology, Institute of Experimental Psychology, University of Düsseldorf, Düsseldorf, Germany \\ ${ }^{6}$ Department of Psychology, University of Freiburg, Freiburg, Germany \\ ${ }^{7}$ Department of Clinical Psychology and Psychotherapy, Institute of \\ Psychology, University of Osnabrück, Osnabrück, Germany}

Acknowledgements The authors are grateful to Robert Richter, MSc, for his help with study selection, and to Hans-Christian Puls, MSc, for his help with coding. They are further grateful to Anja Sembill, for providing the codes from 
the Vocks et $a f^{13}$ meta-analysis for determining inter-rater reliability. They acknowledge support from the German Research Foundation (DFG) and University of Leipzig within the program of Open Access Publishing.

Contributors $\mathrm{AH}, \mathrm{DP}, \mathrm{RS}, \mathrm{SH}, \mathrm{AK}, \mathrm{RP}, \mathrm{BT}-\mathrm{C}$ and SV were involved in protocol writing. $\mathrm{RS}$ and $\mathrm{AH}$ were involved in study selection, data extraction and disagreement resolution. RS was involved in data entry. DP was involved in data analysis. AH, DP and RS were involved in reporting. AH, SH, AK, RP, BT-C and SV are forming the BED Clinical Guidelines Group, under the auspices of which this meta-analysis is conducted.

Funding This work was supported by funds from the Christina Barz Foundation. AH, DP and RS were supported by grant $01 \mathrm{E} 01501$ from the German Federal Ministry of Education and Research.

Competing interests The authors will give presentations on this topic, with honoraria and travel costs covered by organisers.

Provenance and peer review Not commissioned; externally peer reviewed.

Open Access This is an Open Access article distributed in accordance with the Creative Commons Attribution Non Commercial (CC BY-NC 4.0) license which permits others to distribute, remix, adapt, build upon this work noncommercially, and license their derivative works on different terms, provided the original work is properly cited and the use is non-commercial. See: http:// creativecommons.org/licenses/by-nc/4.0/

\section{REFERENCES}

1. American Psychiatric Association. Diagnostic and Statistical Manual of Mental Disorders 5th Edition (DSM-5). Arlington, VA: American Psychiatric Association, 2013.

2. American Psychiatric Association. Diagnostic and Statistical Manual of Mental Disorders 4th Edition (DSM-IV). Washington DC: American Psychiatric Association, 1994.

3. Kessler RC, Berglund PA, Chiu WT, et al. The prevalence and correlates of binge eating disorder in the World Health Organization World Mental Health Surveys. Biol Psychiatry 2013;73:904-14.

4. Swanson SA, Crow SJ, Le Grange D, et al. Prevalence and correlates of eating disorders in adolescents: results from the National Comorbidity Survey Replication Adolescent Supplement. Arch Gen Psychiatry 2011;68:714-23.

5. Gartlehner G, Hansen RA, Nissman D, et al. A simple and valid tool distinguished efficacy from effectiveness studies. J Clin Epidemiol 2006;59:1040-8.

6. Berkman ND, Brownley KA, Peat CM, et al. Management and outcomes of binge-eating disorder. Comparative effectiveness review No. 160. Rockville, MD: Agency for Healthcare Research and Quality, 2015. http://www.effectivehealthcare.ahrq.gov/reports/final. cfm (accessed 28 Jul, 2016)

7. Beintner I, Jacobi C, Schmidt UH. Participation and outcome in manualized self-help for bulimia nervosa and binge eating disorder - a systematic review and metaregression analysis. Clin Psychol Rev 2014;34:158-76.

8. Brownley KA, Berkman ND, Peat CM, et al. Binge-eating disorder in adults: a systematic review and meta-analysis. Ann Intern Med 2016;165:409-20.

9. Hay PJ, Bacaltchuk J, Stefano S. Psychotherapy for bulimia nervosa and binging. Cochrane Database Syst Rev 2004;(3):CD000562.

10. Hay PPJ, Bacaltchuk J, Stefano S, et al. Psychological treatments for bulimia nervosa and binging. Cochrane Database Syst Rev 2009; (4):CD000562.

11. Stefano SC, Bacaltchuk J, Blay SL, et al. Antidepressants in short-term treatment of binge eating disorder: systematic review and meta-analysis. Eat Behav 2008;9:129-36.

12. Reas DL, Grilo CM. Review and meta-analysis of pharmacotherapy for binge-eating disorder. Obesity (Silver Spring) 2008;16:2024-38.

13. Vocks S, Tuschen-Caffier B, Pietrowsky R, et al. Meta-analysis of the effectiveness of psychological and pharmacological treatments for binge eating disorder. Int J Eat Disord 2010;43:205-17.
14. Brownley KA, Berkman ND, Sedway JA, et al. Binge eating disorder treatment: a systematic review of randomized controlled trials. Int $J$ Eat Disord 2007;40:337-48.

15. da Luz FQ, Hay P, Gibson AA, et al. Does severe dietary energy restriction increase binge eating in overweight or obese individuals? A systematic review. Obes Rev 2015;16:652-65

16. Hay PJ, Claudino AM. Clinical psychopharmacology of eating disorders: a research update. Int J Neuropsychopharmacol 2012;15:209-22.

17. Hay P. A systematic review of evidence for psychological treatments in eating disorders: 2005-2012. Int J Eat Disord 2013;46:462-9.

18. McElroy SL, Guerdjikova Al, Mori N, et al. Pharmacological management of binge eating disorder: current and emerging treatment options. Ther Clin Risk Manag 2012;8:219-41.

19. Reas DL, Grilo CM. Pharmacological treatment of binge eating disorder: update review and synthesis. Expert Opin Pharmacother 2015;16:1463-78.

20. lacovino JM, Gredysa DM, Altman M, et al. Psychological treatments for binge eating disorder. Curr Psychiatry Rep 2012;14:432-46.

21. Wilson GT. Treatment of binge eating disorder. Psychiatr Clin North Am 2011;34:773-83.

22. Wilson GT, Zandberg LJ. Cognitive-behavioral guided self-help for eating disorders: effectiveness and scalability. Clin Psychol Rev 2012;32:343-57.

23. Fornaro M, Solmi M, Perna G, et al. Lisdexamfetamine in the treatment of moderate-to-severe binge eating disorder in adults: systematic review and exploratory meta-analysis of publicly available placebo-controlled, randomized clinical trials. Neuropsychiatr Dis Treat 2016;12:1827-36.

24. Grilo CM, Reas DL, Mitchell JE. Combining pharmacological and psychological treatments for binge eating disorder: current status, limitations, and future directions. Curr Psychiatry Rep 2016;18:55

25. Wilson GT, Wilfley DE, Agras WS, et al. Psychological treatments of binge eating disorder. Arch Gen Psychiatry 2010;67:94-101.

26. Grilo CM, Crosby RD, Wilson GT, et al. 12-month follow-up of fluoxetine and cognitive behavioral therapy for binge eating disorder. $J$ Consult Clin Psychol 2012;80:1108-13.

27. Hilbert A, Bishop ME, Stein RI, et al. Long-term efficacy of psychological treatments for binge eating disorder. Br J Psychiatry 2012;200:232-7.

28. Moher D, Shamseer L, Clarke $M$, et al. Preferred reporting items for systematic review and meta-analysis protocols (PRISMA-P) 2015 statement. Syst Rev 2015;4:1.

29. Stroup DF, Berlin JA, Morton SC, et al. Meta-analysis of observational studies in epidemiology. A proposal for reporting JAMA 2000;283:2008-12.

30. Association of the Scientific Medical Societies. [Arbeitsgemeinschaft der Wissenschaftlichen Medizinischen Fachgesellschaften, AWMF] (2010). S3 Leitlinie Diagnostik und Therapie von Essstörungen [S3 Guidelines of Diagnosis and Treatment of Eating Disorders]. http:// www.awmf.org/leitlinien/detail/ll/051-026.html (accessed $28 \mathrm{Jul}$ 2016).

31. Wilson GT, Fairburn CG. Cognitive treatments for eating disorders. $J$ Consult Clin Psychol 1993;61:261-9.

32. Meany G, Conceição E, Mitchell JE. Binge eating, binge eating disorder and loss of control eating: effects on weight outcomes after bariatric surgery. Eur Eat Disord Rev 2014;22:87-91.

33. Higgins JPT, Green S, eds. Cochrane handbook for systematic reviews of interventions version 5.1.0. [updated March 2011]. The Cochrane Collaboration, 2011. http://www.handbook.cochrane.org (accessed 28 Jul, 2016).

34. Effective Practice and Organisation of Care (EPOC). Suggested risk of bias criteria for EPOC reviews. EPOC resources for review authors. Oslo: Norwegian Knowledge Centre for the Health Services, 2015. http://epoc.cochrane.org/ epoc-specific-resources-review-authors (accessed $28 \mathrm{Jul} 2016$ ).

35. Sterne JA, Hernán MA, Reeves BC, et al. ROBINS-l: a tool for assessing risk of bias in non-randomised studies of interventions. BMJ 2016;355:i4919.

36. O'Neill J, Tabish $\mathrm{H}$, Welch $\mathrm{V}$, et al. Applying an equity lens to interventions: using PROGRESS ensures consideration of socially stratifying factors to illuminate inequities in health. J Clin Epidemiol 2014;67:56-64 